\title{
Interlacing Families IV: Bipartite Ramanujan Graphs of All Sizes *
}

\author{
Adam W. Marcus \\ Crisply \\ Yale University
}

\author{
Daniel A. Spielman \\ Yale University
}

June 1, 2015

\author{
Nikhil Srivastava \\ UC Berkeley
}

\begin{abstract}
We prove that there exist bipartite Ramanujan graphs of every degree and every number of vertices. The proof is based on analyzing the expected characteristic polynomial of a union of random perfect matchings, and involves three ingredients: (1) a formula for the expected characteristic polynomial of the sum of a regular graph with a random permutation of another regular graph, (2) a proof that this expected polynomial is real rooted and that the family of polynomials considered in this sum is an interlacing family, and (3) strong bounds on the roots of the expected characteristic polynomial of a union of random perfect matchings, established using the framework of finite free convolutions introduced recently in [MSS15a].
\end{abstract}

\footnotetext{
${ }^{*}$ This research was partially supported by NSF grant CCF-1111257, an NSF Mathematical Sciences Postdoctoral Research Fellowship, Grant No. DMS-0902962, a Simons Investigator Award to Daniel Spielman, and a MacArthur Fellowship.
} 


\section{Introduction}

Ramanujan graphs are undirected regular graphs whose nontrivial adjacency matrix eigenvalues are asymptotically as small as possible; in other words, they are the optimal spectral expander graphs. In this paper, we prove the existence of bipartite Ramanujan graphs of every degree and every size. We do this by showing that a random $m$-regular bipartite graph, obtained as a union of $m$ random perfect matchings across a bipartition of an even number of vertices, is Ramanujan with nonzero probability. Specifically, we prove that the expected characteristic polynomial of such a random graph has roots concentrated in the appropriate range, and use the method of interlacing families introduced in MSS15b] to deduce that there must be an actual graph whose eigenvalues are no worse than the roots of this polynomial. Infinite families of bipartite Ramanujan graphs were shown in that paper to exist for every degree $m \geq 3$, but it was not known whether they exist for every number of vertices.

The main conceptual and technical contributions of this work and the companion paper [MSS15a] are the following. First, we identify a new class of real-rooted expected characteristic polynomials related to random graphs, and develop new tools for establishing their interlacing properties and analyzing the locations of their roots. These methods are different from those used to study the mixed characteristic polynomials of [MSS15c], and the bounds we obtain are strictly stronger than those produced by the original "barrier method" argument introduced in BSS12] (which is off by a factor of two in this setting). Notably, the expected characteristic polynomials we consider are computable in polynomial time, unlike most other known expected characteristic polynomials. Second, in contrast to previous work, we derive the Ramanujan bound from completely generic considerations involving random orthogonal matrices, in particular making no use of results from algebraic graph theory or number theory.

\subsection{Summary of Results}

Recall that the adjacency matrix $A$ of an $\mathbf{m}$-regular graph on $\mathbf{d}$ vertices 1 has largest eigenvalue $\lambda_{1}(A)=m$, and smallest eigenvalue $\lambda_{d}(A)=-m$ when the graph is bipartite. Following Friedman [Fri08, we will refer to these as the trivial eigenvalues of $A$, and we will call a graph Ramanujan if all of its non-trivial eigenvalues have absolute value at most $2 \sqrt{m-1}$. Such graphs are asymptotically best possible in the sense that a theorem of Alon and Boppana Nil91 tells us that for every $\epsilon>0$, every infinite sequence of $m$-regular graphs must contain a graph with a non-trivial eigenvalue of absolute value at least $2 \sqrt{m-1}-\epsilon$.

Our main theorem is that a union of $m$ random perfect matchings across a bipartition of $2 d$ vertices is Ramanujan with nonzero probability.

Theorem 1.1. Let $P_{1}, \ldots, P_{m}$ be independent uniformly random $d \times d$ permutation matrices, $m \geq 3$. Then, with nonzero probability the nontrivial eigenvalues of

$$
A=\sum_{i=1}^{m}\left[\begin{array}{cc}
0 & P_{i} \\
P_{i}^{T} & 0
\end{array}\right]
$$

are all less than $2 \sqrt{m-1}$ in absolute value.

\footnotetext{
${ }^{1}$ In order to be consistent with our companion paper MSS15a, we will, unconventionally, use $m$ to denote the degree of a graph and $d$ to denote its number of vertices.
} 
We also prove the following non-bipartite version of this theorem, regarding a union of $m$ random perfect matchings on $d$ vertices (not bipartite), with $d$ even.

Theorem 1.2. Let $P_{1}, \ldots, P_{m}$ be independent uniformly random $d \times d$ permutation matrices, $d$ even, $m \geq 3$. Let $M$ be the adjacency matrix of any fixed perfect matching on $d$ vertices. Then with nonzero probability:

$$
\lambda_{2}\left(\sum_{i=1}^{m} P_{i} M P_{i}^{T}\right)<2 \sqrt{m-1} .
$$

Since we only prove nonzero bounds on the probabilities, the nonbipartite theorem is a logical consequence of the bipartite one. We describe it here because its proof is substantially easier and contains most of the main ideas. Note that Theorem 1.2 does not produce Ramanujan graphs because it does not guarantee any control of the least eigenvalue $\lambda_{d}$.

We remark that as they are unions of independent matchings, the graphs we produce may have multiple edges between two vertices. Thus, they are strictly speaking multigraphs, and do not subsume the previous results if one insists on simple graphs. However, it seems that it should be more difficult to construct Ramanujan graphs with multiedges than without. Like [MSS15b], this paper establishes existence but does not give a polynomial time construction of Ramanujan graphs.

\subsection{Related Work}

Infinite families of Ramanujan graphs were first shown to exist for $m=p+1, p$ a prime, in the seminal work of Margulis and Lubotzky-Phillips-Sarnak [Mar88, LPS88]. The graphs they produce are Cayley graphs and can be constructed very efficiently, and their analysis relies on deep results from number theory, which is responsible for the "Ramanujan" nomenclature. Friedman [Fri08] showed that a random $m$-regular graph is almost Ramanujan: specifically, that a union of $m$ perfect matchings has non-trivial eigenvalues bounded by $2 \sqrt{m-1}+\epsilon$ with high probability, for every $\epsilon>0$. More recently, in MSS15b], we proved the existence of infinite families of $m$-regular bipartite Ramanujan graphs for every $m \geq 3$ by proving (part of) a conjecture of Bilu and Linial [BL06] regarding the existence of good 2-lifts of regular graphs. Prior to the present paper, it was unknown if there exist Ramanujan graphs of every number of vertices. We refer the reader to [HLW06] and [MSS15b] for a more detailed discussion of Ramanujan graphs and 2-lifts.

\subsection{Outline of the Paper}

The proofs of both of our theorems follow the same strategy and consist of three steps. In each step we present the simpler non-bipartite case first, and then indicate the modifications required for the bipartite case.

First, we show that the expected characteristic polynomials of the random graphs we are interested in are real rooted and come from interlacing families (reviewed in Section 2.1), which reduces our existence theorems to analyzing the roots of these polynomials. This is achieved in Section 3 by decomposing the random permutations used to generate these expected polynomials into swaps acting on two vertices at a time, and showing that such swaps correspond to linear transformations which preserve real-rootedness properties of certain multivariate polynomials. Theorem 3.3 implies that if $A$ and $B$ are symmetric matrices, then the expected characteristic polynomial of $A+P B P^{T}$ is real rooted for a random permutation matrix $P$. We remark that this argument is completely elementary and self-contained, and unlike MSS15b, MSS15c does not appeal to any 
results from the theory of real stable or hyperbolic polynomials. In the process, we introduce a class of "determinant-like" polynomials which may be of independent interest.

Next, in Section 4 we derive a closed-form formula for the expected characteristic polynomial of a sum of randomly permuted regular graphs. We begin by proving that the expected characteristic polynomials over random permutations can be replaced by expected characteristic polynomials over random orthogonormal matrices. This may be seen as a quadrature or derandomization statement, which says that these characteristic polynomials are not able to distinguish between the set of permutation matrices and the set of orthogonal matrices; essentially this happens because determinants are multilinear, which causes certain restrictions of them to have very low degree Fourier coefficients. This component of the proof may also be of independent interest.

Finally, we appeal to machinery developed in our companion paper [MSS15a], which studies the structure of expected characteristic polynomials over random orthogonal matrices. In particular, such polynomials may be expressed crisply in terms of a simple (and explicitly computable) convolution operation on characteristic polynomials, which we call the finite free additive convolution. In this framework, the characteristic polynomial of a union of $m$ random matchings is simply the $m$-wise convolution of the characteristic polynomial of a single matching. By applying strong bounds on the roots of these convolutions derived in [MSS15a, we obtain the desired Ramanujan bound of $2 \sqrt{m-1}$. The requisite material regarding free convolutions is introduced in Sections 2.2 and 2.3 .

These three ingredients are combined in Section 5 to complete the proofs of Theorems 1.1 and 1.2

\section{Preliminaries}

\subsection{Interlacing Families}

We recall the following theorem from [MSS15c], stated here in the slightly different language of product distributions.

Theorem 2.1 (Interlacing Families). Suppose $\left\{f_{\omega}(x)\right\}_{\omega \in\{0,1\}^{m}}$ is a family of real-rooted polynomials of the same degree $n$ with positive leading coefficient, such that

$$
E_{\mu}(x):=\mathbb{E}_{\omega \sim \mu} f_{\omega}(x)
$$

is real-rooted for every product distribution $\mu=\mu_{1} \otimes \ldots \otimes \mu_{m}$ on $\Omega=\{0,1\}^{m}$. Then for every $k=1, \ldots, n$ and every such $\mu$, there is some $\omega_{0} \in \Omega$ such that

$$
\lambda_{k}\left(f_{\omega_{0}}\right) \leq \lambda_{k}\left(E_{\mu}\right),
$$

where $\lambda_{k}$ denotes the $k$ th largest root of a real-rooted polynomial.

For real rooted polynomials $f$ and $g$, we write $g \longrightarrow f$ if the roots of $f$ and $g$ interlace and the largest root of $f$ is at least as big as the largest root of $g$. We will use the following elementary facts about interlacing and real-rootedness, which may be found in [Fis08.

Lemma 2.2. If $g$ has degree one less than $f$ and both are real-rooted, then

1. $g \longrightarrow f$ if and only if $f+\alpha g$ is real-rooted for all $\alpha \in \mathbb{R}$ 


\section{2. $g \longrightarrow f$ implies that $f \longrightarrow f-g$.}

If $f_{1}$ and $f_{2}$ are monic and real-rooted of the same degree, then they have a common interlacing if and only if $f_{1}+\alpha f_{2}$ is real-rooted for all $\alpha \geq 0$.

\subsection{Finite Free Convolutions of Polynomials}

To analyze the expected characteristic polynomials of the random graphs we consider, we will need the notion of a finite free convolution of two polynomials, developed in our companion paper MSS15a. We denote the characteristic polynomial of a matrix by:

$$
\chi_{x}(A):=\operatorname{det}(x I-A) .
$$

Definition 2.3 (Symmetric Additive Convolution). Let $p(x)=\chi_{x}(A)$ and $q(x)=\chi_{x}(B)$ be two real-rooted polynomials, for some symmetric $d \times d$ matrices $A$ and $B$. The symmetric additive convolution of $p$ and $q$ is defined as:

$$
p(x) \boxplus_{d} q(x)=\underset{Q}{\mathbb{E}} \chi_{x}\left(A+Q B Q^{T}\right),
$$

where the expectation is taken over random orthogonal matrices $Q$ sampled according to the Haar measure on $O(d)$, the group of $d$-dimensional orthonormal matrices.

Note that this is a well-defined operation on polynomials because the distribution of the eigenvalues of $A+Q B Q^{T}$ depends only on the eigenvalues of $A$ and the eigenvalues of $B$, which are the roots of $p$ and $q$.

Definition 2.4 (Asymmetric Additive Convolution). Let $p(x)=\chi_{x}\left(A A^{T}\right)$ and $q(x)=\chi_{x}\left(B B^{T}\right)$ be two real-rooted polynomials with nonnegative roots, for some arbitrary (not necessarily symmetric) $d \times d$ matrices $A$ and $B$. The asymmetric additive convolution of $p$ and $q$ is defined as

$$
p(x) \boxplus_{d} q(x)=\underset{Q, R}{\mathbb{E}} \chi_{x}\left(\left(A+Q B R^{T}\right)\left(A+Q B R^{T}\right)^{T}\right),
$$

where $Q$ and $R$ are independent random orthogonal matrices sampled uniformly from $O(d)$.

When dealing with a possibly asymmetric $d \times d$ matrix $M$, we will frequently consider the dilation

$$
\left[\begin{array}{cc}
0 & M \\
M^{T} & 0
\end{array}\right]
$$

which is by construction a symmetric $2 d \times 2 d$ matrix. We will refer to a matrix of this type as a bipartite matrix. It is easy to see that its eigenvalues are symmetric about 0 and are equal to $\pm \lambda_{1}\left(M M^{T}\right)^{1 / 2}, \ldots, \pm \lambda_{d}\left(M M^{T}\right)^{1 / 2}$, i.e., in absolute value to the singular values of $M$. This correspondence also gives the useful identity

$$
\mathbb{S} \chi_{x}\left(M M^{T}\right)=\chi_{x}\left(\left[\begin{array}{cc}
0 & M \\
M^{T} & 0
\end{array}\right]\right)
$$

where the operator $\mathbb{S}$ is defined by

$$
(\mathbb{S} p)(x):=p\left(x^{2}\right) .
$$


With this notation in hand, we can alternately express the asymmetric additive convolution as

$$
\mathbb{S}\left(p(x) \boxplus_{d} q(x)\right)=\underset{Q, R}{\mathbb{E}} \chi_{x}\left(\left[\begin{array}{cc}
0 & A \\
A^{T} & 0
\end{array}\right]+\left[\begin{array}{cc}
Q & 0 \\
0 & R
\end{array}\right]\left[\begin{array}{cc}
0 & B \\
B^{T} & 0
\end{array}\right]\left[\begin{array}{cc}
Q & 0 \\
0 & R
\end{array}\right]^{T}\right)
$$

Explicit, polynomial time computable formulas for the additive convolutions in terms of the coefficients of $p$ and $q$ may be found in Theorems 1.1 and 1.3 of [MSS15a. For this work, we only require the following important consequences of these formulas, also established in [MSS15a. We will occasionally drop the subscripts in $\boxplus_{d}$ and $\Psi_{d}$ when it is clear from the context.

Lemma 2.5 (Properties of $\boxplus$ and $\boxplus \boxplus)$. $\quad$ 1. If $p(x)$ and $q(x)$ are real-rooted then $p(x) \boxplus_{d} q(x)$ is also real-rooted.

2. If $p(x)$ and $q(x)$ are real-rooted with all roots nonnegative, then $p(x) \uplus^{+} \oplus_{d} q(x)$ is also realrooted with all roots nonnegative.

3. The operations $\boxplus_{d}$ and $\Psi_{d}$ are bilinear (in the coefficients of the polynomials on which they operate) and associative.

Proof. (1) and (2) are Theorems 1.2 and 1.4 of [MSS15a, and bilinearity follows immediately from Theorems 1.1 and 1.3 of MSS15a. To see associativity, let $p(x)=\chi_{x}(A), q(x)=\chi_{x}(B)$ and $r(x)=\chi_{x}(C)$, and observe that

$$
\begin{aligned}
(p(x) \boxplus q(x)) \boxplus r(x) & =\left(\underset{Q}{\mathbb{E}} \underset{R}{\mathbb{E}} \chi_{x}\left(Q A Q^{T}+R B R^{T}\right)\right) \boxplus \chi_{x}(C) \\
& =\underset{Q}{\mathbb{R}} \underset{R}{\mathbb{E}}\left(\chi_{x}\left(Q A Q+R B R^{T}\right) \boxplus \chi_{x}(C)\right) \quad \text { by bilinearity } \\
& =\underset{Q}{\mathbb{E}} \underset{R}{\mathbb{E}} \chi_{x}\left(Q A Q+R B R^{T}+W C W^{T}\right),
\end{aligned}
$$

for random orthogonal matrices $Q, R, W$. The same argument shows that this is also equal to $p(x) \boxplus(q(x) \boxplus r(x))$.

An analogous argument using the formula (2) shows that \pm \pm is also associative.

A consequence of the above lemma is that for $m$ matrices $A_{1}, \ldots, A_{m}$, identities such as

$$
\underset{Q_{1}, \ldots, Q_{m}}{\mathbb{E}} \chi_{x}\left(\sum_{i=1}^{m} Q_{i} A_{i} Q_{i}^{T}\right)=\chi_{x}\left(A_{1}\right) \boxplus \chi_{x}\left(A_{2}\right) \boxplus \ldots \boxplus \chi_{x}\left(A_{m}\right)
$$

make sense.

\subsection{Cauchy Transforms}

The device that we use to analyze the roots of finite free convolutions of polynomials is the Cauchy Transform. This is the same (up to normalization) as the Stieltjes Transform and the "Barrier Function" of [BSS12, MSS15b, MSS15c]. 
Definition 2.6 (Cauchy Transform). The Cauchy Transform of a polynomial $p(x)$ with roots $\lambda_{1}, \ldots, \lambda_{d}$ is defined to be the function

$$
\mathcal{G}_{p}(x)=\frac{1}{d} \sum_{i=1}^{d} \frac{1}{x-\lambda_{i}}=\frac{1}{d} \frac{p^{\prime}(x)}{p(x)} .
$$

We define the inverse Cauchy Transform of $p$ to be

$$
\mathcal{K}_{p}(w)=\max \left\{x: \mathcal{G}_{p}(x)=w\right\} .
$$

Note that the Cauchy transform has poles at the roots of $p$, and when all the roots $\lambda_{i}$ of $p$ are real, $\mathcal{G}_{p}(x)$ is monotone decreasing for $x$ greater than the largest root. Thus, $\mathcal{K}_{p}(w)$ is the unique value of $x$ that is larger than all the $\lambda_{i}$ for which $\mathcal{G}_{p}(x)=w$. In particular, it is an upper bound on the largest root of $p$, and approaches the largest root as $w \rightarrow \infty$.

Our bounds on the expected characteristic polynomials of random graphs are a consequence of the following two theorems, which are proved in MSS15a.

Theorem 2.7 (Theorem 1.7 of [MSS15a]). For real-rooted degree d polynomials $p$ and $q$ and $w>0$,

$$
\mathcal{K}_{p \boxplus_{d} q}(w) \leq \mathcal{K}_{p}(w)+\mathcal{K}_{q}(w)-1 / w .
$$

The above theorem is a strengthening of the univariate barrier function argument for characteristic polynomials introduced in [BSS12]. This may be seen by taking $q(x)=\chi_{x}(B)=x^{d-1}(x-d)$, which corresponds to a rank one matrix $B=v v^{T}$ with trace equal to $d$. It is easy to check that in this case $p(x) \boxplus q(x)=p(x)-p^{\prime}(x)$.

We remark that Theorem 2.7 is inspired by an equality regarding inverse Cauchy transforms of limiting spectral distributions of certain random matrix models arising in Free Probability theory; we refer the interested reader to [MSS15a] for a more detailed discussion. To analyze the case of bipartite random graphs, we will need the corresponding inequality for the asymmetric convolution.

Theorem 2.8 (Theorem 1.8 of [MSS15a]). For degree $d$ polynomials $p$ and $q$ having only nonnegative real roots,

$$
\mathcal{K}_{\mathbb{S}\left(p \mp \mp_{d} q\right)}(w) \leq \mathcal{K}_{\mathbb{S} p}(w)+\mathcal{K}_{\mathbb{S} q}(w)-1 / w
$$

\section{Interlacing for Permutations}

In this section, we show that the expected characteristic polynomials obtained by averaging over certain random permutation matrices form interlacing families. The class of random permutations which have this property are those that are products of independent random swaps, which we now formally define.

Definition 3.1 (Random Swap). A random swap is a matrix-valued random variable which is equal to a transposition of two (fixed) indices $s, t$ with probability $\alpha$ and equal to the identity with probability $(1-\alpha)$, for some $\alpha \in[0,1]$.

Definition 3.2 (Realizability by Swaps). A matrix-valued random variable $P$ supported on permutation matrices is realizable by swaps if there are random swaps $S_{1}, \ldots, S_{N}$ such that the distribution of $P$ is the same as the distribution of the product $S_{N} S_{N-1} \ldots S_{2} S_{1}$. 
For example, we show in Lemma 3.5 below that a uniformly random permutation matrix is realizable by swaps.

The main result of this section is that expected characteristic polynomials over products of random swaps are always real-rooted. These polynomials play a role analogous to that of mixed characteristic polynomials in [MSS15b, MSS15c].

Theorem 3.3. Let $A_{1}, \ldots, A_{m}$ be symmetric $d \times d$ matrices and let $\left\{S_{i j}\right\}_{i \leq m, j \leq N}$ be independent (not necessarily identical) random swaps. Then the expected characteristic polynomial

$$
\mathbb{E} \operatorname{det}\left(t I-\sum_{i=1}^{m}\left(\prod_{j=N}^{1} S_{i j}\right) A_{i}\left(\prod_{j=1}^{N} S_{i j}^{T}\right)\right)
$$

is real-rooted.

An immediate consequence of Theorems 3.3 and 2.1. applied to the family of polynomials indexed by all possible values of the swaps $S_{i j}$, is the following existence result.

Theorem 3.4 (Interlacing Families for Permutations). Suppose $A_{1}, \ldots, A_{m}$ are symmetric $d \times d$ matrices, and $P_{1}, \ldots, P_{m}$ are independent random permutations realizable by swaps. Then, for every $k \leq d$ :

$$
\lambda_{k}\left(\sum_{i=1}^{m} P_{i} A_{i} P_{i}^{T}\right) \leq \lambda_{k}\left(\mathbb{E} \chi_{x}\left(\sum_{i=1}^{m} P_{i} A_{i} P_{i}^{T}\right)\right),
$$

with nonzero probability.

Theorem 3.4 is useful because the uniform distribution on permutations and its bipartite version, which we use to generate our random graphs, are realizable by swaps.

Lemma 3.5. Let $P$ and $S$ be uniformly random $d \times d$ permutation matrices. Both $P$ and $P \oplus S$ are realizable by swaps, where $P \oplus S=\left(\begin{array}{ll}P & 0 \\ 0 & S\end{array}\right)$ is the direct sum of $P$ and $S$.

Proof. We will establish the claim for $P$ first. We proceed inductively. Let $M_{2}$ be a random swap which swaps $e_{1}$ and $e_{2}$ with probability $1 / 2$, and for $k>2$ let

$$
M_{k}=M_{k-1} S_{1 k} M_{k-1},
$$

where $S_{1 k}$ swaps $e_{1}$ and $e_{k}$ with probability $1 / k$.

Let $v=(1,2,3, \ldots, d)^{T}$. By induction, assume that the first $k-1$ coordinates of $M_{k-1} v$ are in uniformly random order; in particular, that $\left(M_{k-1} v\right)(1)$ is a random element of $\{1, \ldots, k-1\}$. This means that:

- With probability $1 / k:\left(M_{k-1} S_{1 k} M_{k-1} v\right)(k)=k$ and the remaining indices contain a random permutation of $\{1, \ldots, k-1\}$.

- With probability $1-1 / k:\left(M_{k-1} S_{1 k} M_{k-1} v\right)(k)$ is a uniformly random element $j \in\{1, \ldots, k-$ $1\}$ and the remaining indices contain a random permutation of $\{1, \ldots, k\} \backslash\{j\}$.

Thus, $M_{k}$ is uniformly random on $\{1, \ldots, k\}$, and by induction $M_{d}=P$.

For $P \oplus S$, we use the above argument to realize $P \oplus I$ and $I \oplus S$ separately and then multiply them. 
The rest of this section is devoted to proving Theorem 3.3. This is achieved by showing that the polynomials in (4) are univariate restrictions of certain nice multivariate polynomials. The relevant notion is the following.

Definition 3.6 (Determinant-like Polynomials). A homogeneous polynomial $P\left(X_{1}, \ldots, X_{m}\right)$ of degree $d$ in the entries of $m$ symmetric $d \times d$ matrices $X_{1}, \ldots, X_{m}$ is called determinant-like if it has the following two properties.

Hyperbolicity. The univariate restrictions

$$
q(t)=P\left(t I-A_{1}, \ldots, t I-A_{m}\right)
$$

are real-rooted for all symmetric $A_{1}, \ldots, A_{m}$.

This condition is known as hyperbolicity of the polynomial $P\left(X_{1}, \ldots, X_{m}\right)$ with respect to the point $(I, I, \ldots, I)$. We do not discuss the notion of hyperbolicity further, since the selfcontained definition above suffices for this paper. We point the interested reader to Pem12] for a detailed discussion of the theory.

Rank-1 Linearity. For every vector $v$, index $i \leq m$, and real number $s$, we have

$$
P\left(X_{1}, X_{2}, \ldots, X_{i}+s v v^{T}, \ldots, X_{m}\right)=P\left(X_{1}, \ldots, X_{m}\right)+s D_{i, v v^{T}} P\left(X_{1}, \ldots, X_{m}\right)
$$

where

$$
D_{i, v v^{T}} P\left(X_{1}, \ldots, X_{m}\right)=\left.\left(\frac{\partial}{\partial s} P\left(X_{1}, \ldots, X_{i}+s v v^{T}, \ldots, X_{m}\right)\right)\right|_{s=0}
$$

is the directional derivative of $P$ in direction $\left(0, \ldots, v v^{T}, \ldots, 0\right)$, where $v v^{T}$ appears in the $i$ th position. Note that $D_{i, v v^{T}} P\left(X_{1}, \ldots, X_{m}\right)$ is homogeneous of degree $d-1$.

An important example of a determinant-like polynomial is the determinant of a sum of matrices:

$$
P\left(X_{1}, \ldots, X_{m}\right)=\operatorname{det}\left(X_{1}+\ldots+X_{m}\right) .
$$

Hyperbolicity follows from the fact that

$$
P\left(t I-A_{1}, \ldots, t I-A_{m}\right)=\operatorname{det}\left(m t I-A_{1}-\ldots-A_{m}\right)
$$

is the characteristic polynomial of a symmetric matrix. Rank-1 linearity can be seen to follow from the invariance of the determinant under change of basis and its linearity with respect to matrix entries. Alternatively, one can prove it by using the matrix determinant lemma, which tells us $\operatorname{det}\left(X_{1}+s v v^{T}+\ldots+X_{m}\right)=\operatorname{det}\left(X_{1}+\ldots+X_{m}\right)+s\left\langle v v^{T}, \operatorname{det}\left(X_{1}+\ldots+X_{m}\right)\left(X_{1}+\ldots+X_{m}\right)^{-1}\right\rangle$.

The crux of the proof of Theorem 3.3 lies in the fact that random swaps define linear operators which preserve the property of being determinant-like.

Lemma 3.7 (Random swaps preserve determinant-likeness). If $P\left(X_{1}, \ldots, X_{m}\right)$ is determinant-like, then for any $i \leq m$ and random swap $S$, the polynomial

$$
\mathbb{E}_{S} P\left(X_{1}, \ldots, S X_{i} S^{T}, \ldots, X_{m}\right)
$$

is determinant-like. 
Before proving this lemma, we record some preliminary facts about determinant-like polynomials.

Lemma 3.8 (Rank-1 updates interlace). Suppose $P\left(X_{1}, \ldots, X_{m}\right)$ is determinant-like. Then for every vector $v$ and symmetric matrices $A_{1}, \ldots, A_{m}$ we have

$$
P\left(t I-A_{1}, \ldots, t I-A_{m}\right) \longrightarrow P\left(t I-A_{1}, \ldots, t I-A_{i}-v v^{T}, \ldots t I-A_{m}\right),
$$

where $\longrightarrow$ denotes interlacing, pointing to the polynomial with the largest root.

Proof. Assume without loss of generality that $i=1$. By rank-1 linearity,

$$
P\left(t I-A_{1}-s v v^{T}, \ldots, t I-A_{m}\right)=P\left(t I-A_{1}, \ldots, t I-A_{m}\right)-s D_{v v^{T}} P\left(t I-A_{1}, \ldots, t I-A_{m}\right) .
$$

By the hyperbolicity of $P$, we know that this is real rooted when viewed as a univariate polynomial in $t$. Since $D_{1, v v^{T}} P$ is of degree one less than $P$, the first part of Lemma 2.2 implies that

$$
D_{1, v v^{T}} P\left(t I-A_{1}, \ldots, t I-A_{m}\right) \longrightarrow P\left(t I-A_{1}, \ldots, t I-A_{m}\right),
$$

which in turn by the second part of Lemma 2.2 gives

$$
\begin{aligned}
P\left(t I-A_{1}, \ldots, t I-A_{m}\right) & \longrightarrow P\left(t I-A_{1}, \ldots, t I-A_{m}\right)-D_{1, v v^{T}} P\left(A_{1}-t I, \ldots, A_{m}-t I\right) \\
& =P\left(t I-A_{1}-v v^{T}, \ldots, t I-A_{m}\right),
\end{aligned}
$$

as desired.

Lemma 3.9 (Permutations preserve rank-1 linearity). (1) If $\Pi$ is a permutation matrix and $P\left(X_{1}, \ldots, X_{m}\right)$ is rank-1 linear then $P\left(\Pi X_{1} \Pi^{T}, X_{2}, \ldots, X_{m}\right)$ is also rank-1 linear. (2) If $P$ and $Q$ are rank-1 linear then so is $P+Q$.

Proof. (1) is true because the set of rank one matrices is invariant under conjugation by permutations. (2) holds because $D_{i, v v^{T}}$ is a linear operator.

We will also need the following elementary observation, which says that random swaps correspond to trace zero rank two updates. This is the structural property which causes interlacing to occur.

Lemma 3.10. If $\sigma$ is a transposition and $A$ is symmetric then $A-\sigma A \sigma^{T}$ has rank 2 and trace 0.

Proof. Assume without loss of generality that $\sigma$ swaps the first two coordinates. Then by symmetry the difference $A-\sigma A \sigma^{T}$ has entries

$$
\left[\begin{array}{ccccc}
a_{11}-a_{22} & a_{12}-a_{21} & a_{13}-a_{23} & a_{14}-a_{24} & \cdots \\
a_{21}-a_{12} & a_{22}-a_{11} & a_{23}-a_{13} & a_{24}-a_{14} & \cdots \\
a_{31}-a_{32} & a_{32}-a_{31} & 0 & \cdots & \\
a_{41}-a_{42} & a_{42}-a_{41} & 0 & \cdots & \\
\ldots & & &
\end{array}\right]=\left[\begin{array}{ccc}
\alpha & \beta & v^{T} \\
\beta & -\alpha & -v^{T} \\
v & -v & 0
\end{array}\right]
$$

for some numbers $\alpha, \beta$ and some column vector $v$ of length $d-2$. If $\alpha \neq \beta$ then the sum of the first two rows is equal to $(c,-c, 0, \ldots, 0)$ for some $c \neq 0$, and every other row is a scalar multiple of this. On the other hand, if $\alpha=\beta$ then the first two rows are linearly dependent, and all of the other rows are multiples of $(1,-1,0, \ldots, 0)$. 
We can now complete the proof of Lemma 3.7

Proof of Lemma 3.7. Assume $P$ is determinant-like, and let $S$ be a random swap, equal to some transposition $\sigma$ with probability $\alpha$ and the identity with probability $(1-\alpha)$. We will show that

$$
Q\left(X_{1}, \ldots, X_{m}\right)=(1-\alpha) P\left(X_{1}, \ldots, X_{m}\right)+\alpha P\left(X_{1}, \ldots, \sigma X_{i} \sigma^{T}, \ldots, X_{m}\right),
$$

is hyperbolic and rank-1 linear. It is clear that $Q\left(X_{1}, \ldots, X_{m}\right)$ is homogeneous since swaps and convex combinations preserve homogeneity. Lemma 3.9 implies that rank-1 linearity is also preserved, so all that remains is hyperbolicity. Assume without loss of generality that $i=1$ and consider any univariate restriction along $(I, I, \ldots, I)$ :

$$
Q\left(t I-A_{1}, \ldots, t I-A_{m}\right)=(1-\alpha) P\left(t I-A_{1}, \ldots, t I-A_{m}\right)+\alpha P\left(t I-\sigma A_{1} \sigma^{T}, \ldots, t I-A_{m}\right) .
$$

We need to show that this has all real roots. Observe that the second polynomial may be written as

$$
P\left(t I-A_{1}-a a^{T}+b b^{T}, \ldots, t I-A_{m}\right),
$$

for some vectors $a$ and $b$, since $\sigma A_{1} \sigma^{T}-A_{1}$ is rank two and trace zero by Lemma 3.10. Since $P$ is determinant-like, Lemma 3.8 tells us that

$$
P\left(t I-A_{1}+b b^{T}, \ldots, t I-A_{m}\right) \longrightarrow P\left(t I-A_{1}-a a^{T}+b b^{T}, \ldots, t I-A_{m}\right)
$$

and

$$
P\left(t I-A_{1}+b b^{T}, \ldots, t I-A_{m}\right) \longrightarrow P\left(t I-A_{1}, \ldots, t I-A_{m}\right),
$$

whence the two polynomials on the right hand side of (5) have a common interlacing. Lemma 2.2 then implies that their convex combination must be real-rooted, and the claim is proved.

Applying Lemma 3.7 inductively yields Theorem 3.3.

Proof of Theorem 3.3. Applying Lemma $3.7 n N$ times (once for every swap $S_{i j}$ ) starting with $P\left(X_{1}, \ldots, X_{m}\right)=\operatorname{det}\left(\sum_{i} X_{i}\right)$ tells us that

$$
\mathbb{E}_{S_{1 N}} \ldots \mathbb{E}_{S_{11}} \mathbb{E}_{S_{2 N}} \ldots \mathbb{E}_{S_{n 1}} \operatorname{det}\left(\sum_{i=1}^{n}\left(\prod_{j=N}^{1} S_{i j}\right) X_{i}\left(\prod_{j=1}^{N} S_{i j}^{T}\right)\right)
$$

is determinant-like. Considering the restriction $X_{i}=(t / m) I-A_{i}$ finishes the proof.

\section{Quadrature}

In this section, we show that the expected characteristic polynomials we are interested in are free convolutions of the characteristic polynomials of perfect matchings, after the trivial eigenvalues corresponding to the all ones vector are removed. This gives us explicit formulas for these polynomials, and more importantly (since we understand the behavior of roots under free convolutions) a way of bounding their roots. We begin by showing how to do this for the symmetric case, which is more transparent and contains all the main ideas. In Section 4.2 we derive the result for the bipartite case as a corollary of the result for the symmetric case. 


\subsection{Quadrature for Symmetric Matrices}

The following theorem gives an explicit formula for the expected characteristic polynomial of the sum of two symmetric matrices with constant row sums when the rows and columns of one of the matrices is randomly permuted. This can be used to compute the expected characteristic polynomial of the Laplacian matrix of the sum of two graphs when one is randomly permuted. In this paper, we use the result to compute the expected characteristic polynomial of the adjacency matrix when both graphs are regular.

Theorem 4.1. Suppose $A$ and $B$ are symmetric $d \times d$ matrices with $A \mathbf{1}=a \mathbf{1}$ and $\mathbf{1}=b \mathbf{1}$. Let $\chi_{x}(A)=(x-a) p(x)$ and $\chi_{x}(B)=(x-b) q(x)$. Then,

$$
\mathbb{E}_{P \chi_{x}}\left(A+P B P^{T}\right)=(x-(a+b)) p(x) \boxplus_{d-1} q(x),
$$

where $P$ is a uniformly random permutation.

We begin by writing (6) in a more concrete form. Observe that all of the matrices $A, B, P$ have 1 as a left and right eigenvector, which means that there is an orthogonal change of basis $V$ (for concreteness, mapping 1 to the standard basis vector $e_{n}$ ) that simultaneously block diagonalizes all of them:

$$
V A V^{T}=\hat{A} \oplus a, \quad V B V^{T}=\hat{B} \oplus b, \quad V P V^{T}=\hat{P} \oplus 1,
$$

where $\hat{A} \oplus a$ denotes the direct sum

$$
\left[\begin{array}{cc}
\hat{A} & 0 \\
0 & a
\end{array}\right]
$$

Since the determinant is invariant under change of basis, we may write

$$
\begin{aligned}
\mathbb{E}_{P} \operatorname{det}\left(x I-A-P B P^{T}\right) & =\mathbb{E}_{P} \operatorname{det}\left(x I-V A V^{T}-\left(V P V^{T}\right)\left(V B V^{T}\right)\left(V P^{T} V^{T}\right)\right) \\
& =\mathbb{E}_{\hat{P}} \operatorname{det}\left(x I-(\hat{A} \oplus a)-(\hat{P} \oplus 1)(\hat{B} \oplus b)\left(\hat{P}^{T} \oplus 1\right)\right) \\
& =(x-a-b) \mathbb{E}_{\hat{P}} \operatorname{det}\left(x I-\hat{A}-\hat{P} \hat{B} \hat{P}^{T}\right) .
\end{aligned}
$$

Notice also that $p(x)=\chi_{x}(\hat{A})$ and $q(x)=\chi_{x}(\hat{B})$, so

$$
p(x) \boxplus q(x)=\mathbb{E}_{Q} \operatorname{det}\left(x I-\hat{A}-Q \hat{B} Q^{T}\right),
$$

where $Q$ is a (Haar) random $(d-1) \times(d-1)$ orthogonal matrix. Thus, (6) is equivalent to showing that

$$
\mathbb{E}_{\hat{P}} \operatorname{det}\left(x I-\hat{A}-\hat{P} \hat{B} \hat{P}^{T}\right)=\mathbb{E}_{Q} \operatorname{det}\left(x I-\hat{A}-Q \hat{B} Q^{T}\right),
$$

for all $(d-1) \times(d-1)$ symmetric matrices $\hat{A}, \hat{B}$. Note that for any permutation $P$, the orthogonal transformation $\hat{P}$ correspondingly permutes $\hat{e_{1}}, \ldots, \hat{e_{n}}$, the projections orthogonal to 1 of the standard basis vectors $e_{1}, \ldots, e_{d}$, embedded in $\mathbb{R}^{d-1}$. Since these are the vertices of a regular simplex with $d$ vertices in $\mathbb{R}^{d-1}$ centered at the origin, we interpret the $\hat{P}$ as elements of the symmetry group of this simplex. We denote this subgroup of $O(d-1)$ by $A_{d-1}$.

Since there is no longer any assumption on $\hat{A}, \hat{B}$ other than symmetry, we may absorb the $x I$ term into $\hat{A}$ in (9), and we see that it is sufficient to establish the following. 
Theorem 4.2 (Quadrature Theorem). For symmetric $d \times d$ matrices $A$ and $B$,

$$
\underset{P \in A_{d}}{\mathbb{E}} \operatorname{det}\left(A+P B P^{T}\right)=\underset{Q \in O(d)}{\mathbb{E}} \operatorname{det}\left(A+Q B Q^{T}\right)
$$

It is easy to see that the theorem will follow if we can show that the left hand side of (10) is invariant under right multiplication of $P$ by orthogonal matrices.

Lemma 4.3 (Invariance Implies Quadrature). Let $f$ be a function from $O(d)$ to $\mathbb{R}$ and let $H$ be $a$ finite subgroup of $O(d)$. If

$$
\underset{P \in H}{\mathbb{E}} f(P)=\underset{P \in H}{\mathbb{E}} f\left(P Q_{0}\right)
$$

for all $Q_{0} \in O(d)$, then

$$
\underset{P \in H}{\mathbb{E}} f(P)=\underset{Q \in O(d)}{\mathbb{E}} f(Q)
$$

where $Q$ is chosen according to Haar measure and $P$ is uniform on $H$.

Proof.

$$
\begin{aligned}
\underset{Q \in O(d)}{\mathbb{E}} f(Q) & =\underset{Q \in O(d)}{\mathbb{E}} \underset{P \in H}{\mathbb{E}} f(P Q)=\underset{P \in H}{\mathbb{E}} \underset{Q \in O(d)}{\mathbb{E}} f(P Q) \\
& =\underset{P \in H}{\mathbb{E}} \underset{Q \in O(d)}{\mathbb{E}} f(P)=\underset{P \in H}{\mathbb{E}} f(P),
\end{aligned}
$$

as desired.

We will prove Theorem 4.2 by showing that $f(P)=\operatorname{det}\left(A+P B P^{T}\right)$ satisfies (11). We will achieve this by demonstrating that $f$ is invariant under certain elementary orthogonal transformations acting on 3-faces of the regular simplex, which generate all orthogonal transformations. Let us fix some notation to precisely describe these elementary transformations.

Given three vertices $\hat{e}_{i}, \hat{e_{j}}, \hat{e_{k}}$ of the regular simplex, let $A_{i, j, k}$ denote the subgroup of $A_{d}$ consisting of permutations of $\hat{e}_{i}, \hat{e_{j}}, \hat{e_{k}}$ which leave all of the other vertices fixed. Let $O_{i, j, k}$ denote the subgroup of $O(d)$ acting on the two dimensional linear subspace parallel to the affine subspace through the three vertices, and leaving the orthogonal subspace fixed. Note that $A_{i, j, k}$ is a subgroup of $O_{i, j, k}$, and that these groups are isomorphic to $A_{2}$ and $O(2)$, respectively.

The heart of the proof lies in the following lemma, which implies by Lemma 4.3 that the polynomials we are interested in are not able to distinguish between the uniform distributions on $A_{2}$ and $O(2)$. The reason for this is that these polynomials have very low degree (at most two) in the entries of any orthogonal matrix $Q$ acting on a two-dimensional subspace, a fact which is essentially a consequence of the multilinearity of the determinant. The argument below is similar to the proof of Lemma 2.7 in MSS15a].

Lemma 4.4 (Invariance for $A_{2}$ ). If $A$ and $B$ are symmetric $d \times d$ matrices, then for every $Q_{0} \in$ $O(2)$,

$$
\underset{P \in A_{2}}{\mathbb{E}} \operatorname{det}\left(A+\left(P \oplus I_{d-2}\right) B\left(P \oplus I_{d-2}\right)^{T}\right)=\underset{P \in A_{2}}{\mathbb{E}} \operatorname{det}\left(A+\left(P Q_{0} \oplus I_{d-2}\right) B\left(P Q_{0} \oplus I_{d-2}\right)^{T}\right) .
$$


Proof. Let $S O(2)$ be the subgroup of $O(2)$ consisting of rotation matrices

$$
R_{\theta}=\left[\begin{array}{cc}
\cos \theta & \sin \theta \\
-\sin \theta & \cos \theta
\end{array}\right]
$$

and let $Z_{3}$ be the subgroup of $A_{2}$ consisting of the three rotations $R_{\tau}, \tau \in T:=\{0,2 \pi / 3,4 \pi / 3\}$. We begin by showing that

$$
\underset{P \in Z_{3}}{\mathbb{E}} \operatorname{det}\left(A+(P \oplus I) B(P \oplus I)^{T}\right)=\underset{P \in Z_{3}}{\mathbb{E}} \operatorname{det}\left(A+\left(P R_{\theta} \oplus I\right) B\left(P R_{\theta} \oplus I\right)^{T}\right),
$$

for every $\theta$, where $I$ is the $(d-2)$-dimensional identity. Since the elements of $Z_{3}$ are themselves rotations, we can rewrite thrice the right hand side of (14) as

$$
\begin{aligned}
& \sum_{\tau \in T} \operatorname{det}\left(A+\left(R_{\tau} R_{\theta} \oplus I\right) B\left(R_{\tau} R_{\theta} \oplus I\right)^{T}\right) \\
& =\sum_{\tau \in T} \operatorname{det}\left(A+\left(R_{\tau+\theta} \oplus I\right) B\left(R_{\tau+\theta} \oplus I\right)^{T}\right) \\
& =\sum_{\tau \in T} \sum_{k=-2}^{2} c_{k} e^{i k(\tau+\theta)} \quad \text { for some coefficients } c_{k} \text {, by Lemma 4.5 } \\
& =\sum_{k=-2}^{2} c_{k} e^{i k \theta}\left(e^{i k 0}+e^{i k 2 \pi / 3}+e^{i k 4 \pi / 3}\right) \\
& =3 c_{0} \text { since the terms with }|k|=1,2 \text { vanish. }
\end{aligned}
$$

As this quantity is independent of $\theta$, we can assume $\theta=0$, which gives the left hand side of (14).

To finish the proof, we observe that

$$
\begin{aligned}
\underset{P \in A_{2}}{\mathbb{E} \operatorname{det}\left(A+\left(P \oplus I_{d-2}\right) B\left(P \oplus I_{d-2}\right)^{T}\right)} & =\underset{D \in F}{\mathbb{E}} \underset{P \in Z_{3}}{\mathbb{E}} \operatorname{det}\left(A+(P D \oplus I) B(P D \oplus I)^{T}\right) \\
& =\underset{D \in F}{\mathbb{E}} \underset{P \in Z_{3}}{\mathbb{E}} \operatorname{det}\left(A+(P \oplus I)(D \oplus I) B(D \oplus I)^{T}(P \oplus I)^{T}\right),
\end{aligned}
$$

where $F$ consists of the identity and the reflection across the horizontal axis:

$$
F:=\left\{\left[\begin{array}{ll}
1 & 0 \\
0 & 1
\end{array}\right],\left[\begin{array}{cc}
1 & 0 \\
0 & -1
\end{array}\right]\right\}
$$

and $D$ is chosen uniformly from $F$.

Thus, the left hand side of (13) is invariant under conjugation of $B$ with the matrices $D \oplus I, D \in$ $F$. Since every $Q_{0} \in O(2)$ can be written a $R_{\theta} D$ for some $D \in F$, and we have already established invariance under $R_{\theta} \oplus I$ in (14), the lemma is proved.

Lemma 4.5 (Determinants are Low Degree in Rank 2 Rotations). Let $A, B$ be $d \times d$ matrices and let

$$
\operatorname{det}\left(A+\left(R_{\theta} \oplus I_{d-2}\right) B\left(R_{\theta} \oplus I_{d-2}\right)^{T}\right)=\sum_{k} c_{k} e^{i k \theta} .
$$

Then $c_{k}=0$ for $|k| \geq 3$. 
Proof. Recall that all $2 \times 2$ rotations may be diagonalized as

$$
R_{\theta}=\left[\begin{array}{cc}
\cos \theta & \sin \theta \\
-\sin \theta & \cos \theta
\end{array}\right]=U\left[\begin{array}{cc}
e^{i \theta} & 0 \\
0 & e^{-i \theta}
\end{array}\right] U^{\dagger}
$$

where

$$
U=\frac{1}{\sqrt{2}}\left[\begin{array}{cc}
1 & 1 \\
i & -i
\end{array}\right]
$$

is independent of $\theta$. This implies that $\left(R_{\theta} \oplus I_{d-2}\right)=V D V^{\dagger}$ for diagonal $D$ containing $e^{i \theta}$ and $e^{-i \theta}$ in the upper right $2 \times 2$ block and ones elsewhere, with $V$ independent of $\theta$. Thus, we see that

$$
\begin{aligned}
\operatorname{det}\left(A+\left(R_{\theta} \oplus I_{d-2}\right) B\left(R_{\theta} \oplus I_{d-2}\right)^{T}\right) & =\operatorname{det}\left(A\left(R_{\theta} \oplus I_{d-2}\right)+\left(R_{\theta} \oplus I_{d-2}\right) B\right) \\
& =\operatorname{det}\left(A V D V^{\dagger}+V D V^{\dagger} B\right) \\
& =\operatorname{det}\left(V^{\dagger} A V D+D V^{\dagger} B V\right)
\end{aligned}
$$

Notice that the matrix $M=V^{\dagger} A V D+D V^{\dagger} B V$ depends linearly on $e^{i \theta}, e^{-i \theta}$, and that the $e^{i \theta}$ (resp. $e^{-i \theta}$ ) terms appear only in the first (resp. second) row and column of $M$, respectively. Since each monomial in the expansion of the determinant contains at most one entry from each row and each column and $e^{i \theta} \cdot e^{-i \theta}=1$, this implies that no terms of degree higher than two in $e^{i \theta}$ or $e^{-i \theta}$ appear.

Corollary 4.6 (Invariance for $\left.A_{i, j, k}\right)$. For every $i, j$ and $k$,

$$
\underset{P \in A_{i, j, k}}{\mathbb{E}} \operatorname{det}\left(A+P B P^{T}\right)=\underset{Q \in O_{i, j, k}}{\mathbb{E}} \operatorname{det}\left(A+Q B Q^{T}\right)
$$

Proof. Let $V$ be the orthogonal transformation that maps the affine subspace spanned by the vertices $\hat{e}_{i}, \hat{e}_{j}, \hat{e}_{k}$ to the first two coordinates of $\mathbb{R}^{2}$, with any one vertex mapped to a multiple of $e_{1}$. Conjugation by $V$ maps $A_{i, j, k}$ to $A_{2} \oplus I_{d-2}$ and $O_{i, j, k}$ to $O(2) \oplus I_{d-2}$, abusing notation slightly in the natural way. Since the determinant is invariant under change of basis, Lemma 4.4 tells us that

$$
\begin{aligned}
\underset{P \in A_{i, j, k}}{\mathbb{E}} \operatorname{det}\left(A+P B P^{T}\right) & =\underset{P_{2} \in A_{2}}{\mathbb{E}} \operatorname{det}\left(V A V^{T}+\left(P_{2} \oplus I\right) V B V^{T}\left(P_{2} \oplus I\right)^{T}\right) \\
& =\underset{Q_{2} \in O(2)}{\mathbb{E}} \operatorname{det}\left(V A V^{T}+\left(Q_{2} \oplus I\right) V B V^{T}\left(Q_{2} \oplus I\right)^{T}\right) \\
& =\underset{Q \in O_{i, j, k}}{\mathbb{E}} \operatorname{det}\left(A+Q B Q^{T}\right),
\end{aligned}
$$

as desired.

Lemma $4.7\left(O_{i, j, k}\right.$ generate $\left.O(d)\right)$. Given a regular simplex in $\mathbb{R}^{d}$, the union over $i, j$, and $k$ of $O_{i, j, k}$ generates $O(d)$. In particular, every matrix in $O(d)$ may be written as a product of a finite number of these matrices.

Proof. Let $\Gamma_{h}$ be the subgroup of $O(d)$ generated by $\bigcup_{i, j, k} O_{i, j, k \leq h}$. Let $\hat{e}_{0}, \ldots, \hat{e}_{d}$ be the vertices of the regular simplex. For $1 \leq h \leq d$, let $E_{h}$ be the linear subspace parallel to the affine subspace through $\hat{e}_{0}, \ldots, \hat{e}_{h}$. We will prove by induction on $h$ that $\Gamma_{h}$ contains the action of the orthogonal group on $E_{h}$. The base case is $h=2$, for which $O_{0,1,2}$ is precisely the action of the orthogonal group on $E_{2}$. 
Assuming that we have proved this result for $h-1$, we now prove it for $h$. To this end, let $u_{h}=\hat{e}_{h}$, and let $u_{1}, \ldots, u_{h-1}$ be arbitrary orthonormal vectors in $E_{h}$ that are orthogonal to $u_{h}$. We will prove that for every orthonormal basis $w_{1}, \ldots, w_{h}$ of $E_{h}$, there is a $Q \in \Gamma_{h}$ such that $Q w_{i}=u_{i}$ for $1 \leq i \leq h$.

We first consider the case in which $w_{h}^{T} \hat{e}_{h} \geq 0$. Let $F_{h}$ denote the 2-dimensional affine subspace spanned by $\left\{\hat{e}_{h}, \hat{e}_{h-1}, \hat{e}_{h-2}\right\}$, and observe that there must be a unit vector $p \in E_{h} \cap F_{h}$ with $p^{T} \hat{e}_{h}=$ $w_{h}^{T} \hat{e}_{h}$. This follows because the intersection of $F_{h}$ with the unit sphere in $E_{h}$ is a circle containing $\left\{\hat{e}_{h}, \hat{e}_{h-1}, \hat{e}_{h-2}\right\}, p \mapsto p^{T} \hat{e}_{h}$ is a continuous function, and we have $\hat{e}_{h}^{T} \hat{e}_{h}=1$ and $\hat{e}_{h-1}^{T} \hat{e}_{h}=\hat{e}_{h-2}^{T} \hat{e}_{h}<0$. As $\hat{e}_{h}$ is orthogonal to $E_{h-1}$ and $\hat{e}_{h}$ is invariant under $\Gamma_{h-1}$, the induction hypothesis implies that there must be a $T \in \Gamma_{h-1}$ so that $T w_{h}=p$. Moreover, there is an element $T_{2}$ of $O_{h-2, h-1, h}$ that maps $p$ to $\hat{e}_{h}$. So, their composition $W=T_{2} T$ sends $w_{h}$ to $\hat{e}_{h}$. Since $W$ is orthogonal, it must send $w_{1}, \ldots, w_{h-1}$ to $E_{h-1}$, and so by induction may be composed with a map in $\Gamma_{h-1}$ that sends $W w_{1}, \ldots, W w_{h-1}$ to $u_{1}, \ldots, u_{h-1}$ without moving $\hat{e}_{h}$. The resulting map is the desired $Q$.

In the case that $w_{h}^{T} \hat{e}_{h}<0$, we begin by applying a map in $\Gamma_{h}$ that sends $w_{h}$ to a vector that is orthogonal to $\hat{e}_{h}$ so that we can then apply the previous argument. For example, we can do this by defining $p$ to be one of the two unit vectors in $F_{h}$ with $p^{T} \hat{e}_{h}=-w_{h}^{T} \hat{e}_{h}$. We then apply a map in $\Gamma_{h-1}$ that sends $w_{h}$ to $-p$, and then a map in $O_{h-2, h-1, h}$ that maps $p$, and thus also $-p$, to a vector orthogonal to $\hat{e}_{h}$.

Theorem 4.8 (Invariance for $A_{d}$ ). Let $A$ and $B$ be $d \times d$ matrices, and let

$$
f_{A, B}(Q)=\operatorname{det}\left(A+Q B Q^{T}\right) .
$$

Then, for all $Q_{0} \in O(d)$,

$$
\underset{P \in A_{d}}{\mathbb{E}} f_{A, B}(P)=\underset{P \in A_{d}}{\mathbb{E}} f_{A, B}\left(P Q_{0}\right)
$$

Proof. We will use the fact that

$$
\underset{P \in A_{n}}{\mathbb{E}} f_{A, B}(P)=\underset{P \in A_{d}}{\mathbb{E}} \underset{P_{2} \in A_{i, j, k}}{\mathbb{E}} f_{A, B}\left(P P_{2}\right)=\underset{P \in A_{d}}{\mathbb{E}} \underset{P_{2} \in A_{i, j, k}}{\mathbb{E}} f_{P^{T} A P, B}\left(P_{2}\right)
$$

Applying Corollary 4.6 reveals that for every $Q_{2} \in O_{i, j, k}$,

$$
\begin{aligned}
\underset{P \in A_{d}}{\mathbb{E}} \underset{P_{2} \in A_{i, j, k}}{\mathbb{E}} f_{P^{T} A P, B}\left(P_{2}\right) & =\underset{P \in A_{d}}{\mathbb{E}} \underset{P_{2} \in A_{i, j, k}}{\mathbb{E}} f_{P^{T} A P, B}\left(P_{2} Q_{2}\right) . \\
& =\underset{P \in A_{d}}{\mathbb{E}} \underset{P_{2} \in A_{i, j, k}}{\mathbb{E}} f_{A, B}\left(P P_{2} Q_{2}\right) . \\
& =\underset{P \in A_{d}}{\mathbb{E}} f_{A, B}\left(P Q_{2}\right) .
\end{aligned}
$$

Thus, we conclude that

$$
\underset{P \in A_{d}}{\mathbb{E}} f_{A, B}(P)=\underset{P \in A_{d}}{\mathbb{E}} f_{A, B}\left(P Q_{2}\right)
$$

for every $Q_{2} \in O_{i, j, k}$, for every $i, j, k$.

Let $Q_{0} \in O(d)$. By Lemma 4.7, there is a sequence of matrices $Q_{1}, \ldots, Q_{m}$, each of which is in $O_{i, j, k}$ for some $i, j$ and $k$, so that

$$
Q_{0}=Q_{1} Q_{2} \cdots Q_{m}
$$

By applying the previous equality $m$ times, we obtain

$$
\underset{P \in A_{d}}{\mathbb{E}} f\left(P Q_{0}\right)=\underset{P \in A_{d}}{\mathbb{E}} f\left(P Q_{1} \cdots Q_{m}\right)=\underset{P \in A_{d}}{\mathbb{E}} f(P) .
$$


Proof of Theorem 4.2. Follows from Theorem 4.8 and Lemma 4.3 ,

Proof of Theorem 4.1. Follows from Theorem 4.2, (77), and (8).

We conclude the section by recording the obvious extension of Theorem 4.1 to sums of $m$ matrices.

Corollary 4.9. Let $A_{1}, \ldots, A_{m}$ be symmetric $d \times d$ matrices with $A_{i} \mathbf{1}=a_{i} \mathbf{1}$ and $\chi_{x}\left(A_{i}\right)=(x-$ $\left.a_{i}\right) p_{i}(x)$. Then,

$$
\underset{P_{1}, \ldots, P_{m}}{\mathbb{E}} \chi_{x}\left(\sum_{i=1}^{m} P_{i} A_{i} P_{i}^{T}\right)=\left(x-\sum_{i=1}^{m} a_{i}\right) p_{1}(x) \boxplus \ldots \boxplus p_{m}(x),
$$

where $P_{1}, \ldots, P_{m}$ are independent uniformly random permutation matrices.

Proof. Apply a change of basis so that each $A_{i}=\hat{A}_{i} \oplus a_{i}$, divide out the $\left(x-\sum_{i=1}^{m} a_{i}\right)$ term as in (8), and apply Theorem 4.2 inductively $(m-1)$ times, replacing each $\hat{P}_{i}$ with a random orthogonal $Q_{i}$ (this requires conditioning on the other $\hat{P}_{j}$ and $Q_{j}$, but by independence the distribution of each $\hat{P}_{i}$ is still uniform on $A_{d}$ ). Finally, appeal to the identity (3) to write this as an $m$-wise additive convolution.

\subsection{Quadrature for Bipartite Matrices}

Theorem 4.10. Suppose $A$ and $B$ are (not necessarily symmetric) $d \times d$ matrices such that $A \mathbf{1}=$ $A^{T} \mathbf{1}=a \mathbf{1}$ and $B \mathbf{1}=B^{T} \mathbf{1}=b \mathbf{1}$. Let $\chi_{x}\left(A A^{T}\right)=\left(x-a^{2}\right) p(x)$ and $\chi_{x}\left(B B^{T}\right)=\left(x-b^{2}\right) q(x)$. Then,

$$
\begin{aligned}
\underset{P, S}{\mathbb{E}} \chi_{x}\left(\left[\begin{array}{cc}
0 & A \\
A^{T} & 0
\end{array}\right]+(P \oplus S)\left[\begin{array}{cc}
0 & B \\
B^{T} & 0
\end{array}\right](P \oplus S)^{T}\right) & =\mathbb{S}\left(\left(x-(a+b)^{2}\right) p(x) \oplus \oplus q(x)\right) \\
& =\left(x^{2}-(a+b)^{2}\right) \mathbb{S}(p(x) \oplus \oplus q(x))
\end{aligned}
$$

where $P$ and $S$ are independent uniform random permutation matrices.

As in the nonbipartite case, we begin by applying a change of basis $V$ that isolates the common all ones eigenvector and block diagonalizes our matrices as:

$$
V A V^{T}=\hat{A} \oplus a, V B V^{T}=\hat{B} \oplus b, V P V^{T}=\hat{P} \oplus 1, V S V^{T}=\hat{S} \oplus 1 .
$$

Conjugating the left hand side of (16) by $(V \oplus V)$, we see that it is the same as

$$
\begin{aligned}
& \underset{P, S}{\mathbb{E}} \chi_{x}\left(\left[\begin{array}{cc}
0 & (\hat{A} \oplus a) \\
(\hat{A} \oplus a)^{T} & 0
\end{array}\right]+((\hat{P} \oplus 1) \oplus(\hat{S} \oplus 1))\left[\begin{array}{cc}
0 & (\hat{B} \oplus b) \\
(\hat{B} \oplus b)^{T} & 0
\end{array}\right]((\hat{P} \oplus 1) \oplus(\hat{S} \oplus 1))^{T}\right) \\
& =\underset{P, S}{\mathbb{E}} \chi_{x}\left(\left[\begin{array}{cc}
0 & \left.\hat{A}+\hat{P} \hat{B} \hat{S}^{T} \oplus(a+b)\right)^{T} \\
= & \left(\hat{A}+\hat{P} \hat{B} \hat{S}^{T} \oplus(a+b)\right) \\
P, S & 0
\end{array}\right]\right) \\
& =\left(x^{2}-(a+b)^{2}\right) \underset{P, S}{\mathbb{E}} \mathbb{S} \chi_{x}\left(\left(\hat{A}+\hat{P} \hat{B} \hat{S}^{T}\right)\left(\hat{A}+\hat{P} \hat{B} \hat{S}^{T}\right)^{T}\right) \\
& =\left(x^{2}-(a+b)^{2}\right) \underset{P, S}{\mathbb{E}} \chi_{x}\left(\left[\begin{array}{cc}
0 & \hat{A} \\
\hat{A}^{T} & 0
\end{array}\right]+(\hat{P} \oplus \hat{S})\left[\begin{array}{cc}
0 & \hat{B} \\
\hat{B}^{T} & 0
\end{array}\right](\hat{P} \oplus \hat{S})^{T}\right) .
\end{aligned}
$$


As in the previous section, the matrices $\hat{P}$ and $\hat{S}$ are random elements of the group $A_{d-1}$. Observe that

$$
p(x)=\chi_{x}\left(\hat{A} \hat{A}^{T}\right), \quad \text { and } \quad q(x)=\chi_{x}\left(\hat{B} \hat{B}^{T}\right) .
$$

Recalling from (2) that

$$
\mathbb{S}(p(x) \boxplus \boxplus q(x))=\mathbb{E}_{Q, R \in O(d-1)} \chi_{x}\left(\left[\begin{array}{cc}
0 & A \\
A^{T} & 0
\end{array}\right]+(Q \oplus R)\left[\begin{array}{cc}
0 & B \\
B^{T} & 0
\end{array}\right](Q \oplus R)^{T}\right)
$$

and removing all the $\hat{\imath}$ s as before to ease notation, we see that the conclusion (16) of Theorem 4.10 is implied by the following more general quadrature statement.

Theorem 4.11. For all symmetric $2 d \times 2 d$ matrices $C$ and $D$ :

$$
\underset{P, S \in A_{d}}{\mathbb{E}} \chi_{x}\left(C+(P \oplus S) D(P \oplus S)^{T}\right)=\underset{Q, R \in O(d)}{\mathbb{E}} \chi_{x}\left(C+(Q \oplus R) D(Q \oplus R)^{T}\right) .
$$

This theorem is an immediate consequence of two applications of the following corollary of Theorem 4.2 from the previous section.

Corollary 4.12. If $C$ and $D$ are symmetric $2 d \times 2 d$ matrices,

$$
\underset{P \in A_{d}}{\mathbb{E}} \operatorname{det}\left(C+(P \oplus I) D(P \oplus I)^{T}\right)=\underset{Q \in O(d)}{\mathbb{E}} \operatorname{det}\left(C+(Q \oplus I) D(Q \oplus I)^{T}\right) .
$$

Proof. The proof is identical to the proof of Theorem 4.2, except we replace $P \in A_{d}$ with $P \oplus I$ and $Q \in O(d)$ with $Q \oplus I$ at each step.

Specifically, let

$$
f_{C, D}(Q):=\operatorname{det}\left(C+(Q \oplus I) D(Q \oplus I)^{T}\right) .
$$

Applying Corollary 4.6 as before reveals that for every $i, j, k$ and every $Q_{2} \in O_{i, j, k}$,

$$
\underset{P \in A_{d}}{\mathbb{E}} f_{C, D}(P)=\underset{P \in A_{d}}{\mathbb{E}} \underset{P_{2} \in A_{i, j, k}}{\mathbb{E}} f_{C, D}\left(P P_{2}\right)=\underset{P \in A_{d}}{\mathbb{E}} \underset{P_{2} \in A_{i, j, k}}{\mathbb{E}} f_{C, D}\left(P P_{2} Q_{2}\right)=\underset{P \in A_{d}}{\mathbb{E}} f_{C, D}\left(P Q_{2}\right) .
$$

Since an arbitrary $Q_{0} \in O(d)$ is a finite product of such $Q_{2}$ by Lemma 4.7, this means that

$$
\underset{P \in A_{d}}{\mathbb{E}} f_{C, D}\left(P Q_{0}\right)=\underset{P \in A_{d}}{\mathbb{E}} f_{C, D}(P)
$$

for all $Q_{0} \in O(d)$. Lemma 4.3 completes the proof.

Proof of Theorem 4.11. Since $P$ and $S$ are independent, we have

$$
\begin{aligned}
& \underset{P, S \in A_{d}}{\mathbb{E}} \chi_{x}\left(C+(P \oplus S) D(P \oplus S)^{T}\right) \\
& =\underset{S \in A_{d}}{\mathbb{E}} \underset{P \in A_{d}}{\mathbb{E}} \operatorname{det}\left(x I+C+(P \oplus I)(I \oplus S) D(I \oplus S)^{T}(P \oplus I)^{T}\right) \\
& =\underset{S \in A_{d}}{\mathbb{E}} \underset{Q \in O(d)}{\mathbb{E}} \operatorname{det}\left(x I+C+(Q \oplus I)(I \oplus S) D(I \oplus S)^{T}(Q \oplus I)^{T}\right) \quad \text { by Corollary } 4.12 \\
& =\underset{Q \in O(d)}{\mathbb{E}} \underset{S \in A_{d}}{\mathbb{E}} \operatorname{det}\left(x I+(Q \oplus I)^{T} C(Q \oplus I)+(I \oplus S) D(I \oplus S)^{T}\right) \\
& =\underset{Q \in O(d)}{\mathbb{E}} \underset{R \in O(d)}{\mathbb{E}} \operatorname{det}\left(x I+(Q \oplus I)^{T} C(Q \oplus I)+(I \oplus R) D(I \oplus R)^{T}\right) \quad \text { by Corollary 4.12 } \\
& =\underset{Q, R \in O(d)}{\mathbb{E}} \operatorname{det}\left(x I+C+(Q \oplus R) D(Q \oplus R)^{T}\right),
\end{aligned}
$$

as desired. 
Proof of Theorem 4.10. Follows from Theorem 4.11, (18), and (19).

As before, Theorem 4.10 extends effortlessly to the case of many matrices.

Corollary 4.13. If $A_{1}, \ldots, A_{m}$ are matrices with $A_{i} \mathbf{1}=A_{i}^{T} \mathbf{1}=a_{i}$ and $\chi_{x}\left(A_{i} A_{i}^{T}\right)=\left(x-a_{i}^{2}\right) p_{i}(x)$, then

$$
\begin{aligned}
& \underset{P_{1}, \ldots, P_{m}, S_{1}, \ldots, S_{m}}{\mathbb{E}} \chi_{x}\left(\sum_{i=1}^{m}\left(P_{i} \oplus S_{i}\right)\left[\begin{array}{cc}
0 & A_{i} \\
A_{i}^{T} & 0
\end{array}\right]\left(P_{i} \oplus S_{i}\right)^{T}\right) \\
& =\left(x^{2}-\left(\sum_{i} a_{i}\right)^{2}\right) \mathbb{S}\left[p_{1}(x) \boxplus \oplus \ldots \oplus \oplus p_{m}(x)\right],
\end{aligned}
$$

where the $P_{i}$ and $S_{i}$ are independent uniformly random permutations.

We omit the proof, which is identical to the proof of Corollary 4.9 .

\section{$5 \quad$ Ramanujan Graphs}

In this section, we combine the Cauchy transform, interlacing, and quadrature results of the previous sections to establish our main Theorems 1.1 and 1.2

Proof of Theorem 1.2. Let $M$ be the adjacency matrix of a fixed perfect matching on $d$ vertices, with $d$ even. Since the uniform distribution on permutations is realizable by swaps (Lemma 3.5), Theorem 3.4 tells us that with nonzero probability:

$$
\lambda_{2}\left(\sum_{i=1}^{d} P_{i} M P_{i}^{T}\right) \leq \lambda_{2}\left(\mathbb{E} \chi_{x}\left(\sum_{i=1}^{m} P_{i} M P_{i}^{T}\right)\right) .
$$

Corollary 4.9 reveals that the polynomial in the right-hand expression may be written as an $m$-wise symmetric additive convolution 2

$$
E(x):=\underset{P_{1}, \ldots, P_{m}}{\mathbb{E}} \chi_{x}\left(\sum_{i=1}^{m} P_{i} A_{i} P_{i}^{T}\right)=(x-m) p(x) \boxplus_{d-1} \ldots \boxplus_{d-1} p(x) \quad(m \text { times }),
$$

where

$$
p(x)=\frac{\chi_{M}(x)}{x-1}=(x-1)^{d / 2-1}(x+1)^{d / 2},
$$

is the characteristic polynomial of a single matching with the trivial root at 1 removed. Our goal is therefore to bound the largest root of $p(x) \boxplus \ldots \boxplus p(x)$, which is the second largest root of $E(x)$.

We will do this using the inverse Cauchy transform described in Section 2.3. The Cauchy transform of $p(x)$ is given by

$$
\mathcal{G}_{p}(x)=\frac{d / 2-1}{d-1} \frac{1}{x-1}+\frac{d / 2}{d-1} \frac{1}{x+1} .
$$

\footnotetext{
${ }^{2}$ We remark that this formula works for arbitrary regular adjacency matrices $A_{i}$, as mentioned in the abstract.
} 
Notice that for every $x>1$, putting the trivial root at 1 back only increases the Cauchy transform:

$$
\mathcal{G}_{p}(x)<\frac{d / 2}{d} \frac{1}{x-1}+\frac{d / 2}{d} \frac{1}{x+1}=\frac{x}{x^{2}-1}=\mathcal{G}_{\chi(M)}(x) .
$$

Since both functions are decreasing for $x>1$, this implies that the inverse Cauchy transform of $p$ is upper bounded by that of $\chi(M)$ :

$$
\mathcal{K}_{p}(w)<\mathcal{K}_{\chi(M)}(w)
$$

for every $w>0$.

Applying the convolution inequality in Theorem $2.7(m-1)$ times yields the following upper bound on the inverse Cauchy transform of the $m$-wise convolution of interest.

$$
\mathcal{K}_{p \boxplus \ldots \boxplus p}(w) \leq m \cdot \mathcal{K}_{p}(w)-\frac{m-1}{w}<m \cdot \mathcal{K}_{\chi(M)}(w)-\frac{m-1}{w} .
$$

Recalling from (21) that

$$
\mathcal{K}_{\chi(M)}(w)=x \Longleftrightarrow w=\frac{x}{x^{2}-1},
$$

the right hand side of (22) may be written as

$$
m x-\frac{m-1}{w}=m x-\frac{(m-1)\left(x^{2}-1\right)}{x}=\frac{x^{2}+(m-1)}{x},
$$

which is easily seen to be minimized at $x=\sqrt{m-1}$ with value $2 \sqrt{m-1}$. Thus, the second largest root of $E(x)$ is at most $2 \sqrt{m-1}$.

Proof of Theorem 1.1. Let

$$
M=\left[\begin{array}{cc}
0 & I \\
I^{T} & 0
\end{array}\right]
$$

be the adjacency matrix of a perfect matching on $2 d$ vertices, across the natural bipartition. Then, for independent uniformly random $d \times d$ permutation matrices $P_{1}, \ldots, P_{m}, S_{1}, \ldots, S_{m}$, the random matrix

$$
A=\sum_{i=1}^{m}\left(P_{i} \oplus S_{i}\right) M\left(P_{i} \oplus S_{i}\right)^{T}=\sum_{i=1}^{m}\left[\begin{array}{cc}
0 & \left(P_{i} S_{i}^{T}\right) \\
\left(P_{i} S_{i}^{T}\right)^{T} & 0
\end{array}\right]
$$

is the adjacency matrix of a union of $m$ random matchings across the same bipartition. Since the distribution of the $\left(P_{i} \oplus S_{i}\right)$ is realizable by swaps (Lemma 3.5), Theorem 3.4 implies that

$$
\lambda_{2}(A) \leq \lambda_{2}\left(\mathbb{E} \chi_{x}\left(\sum_{i=1}^{m}\left(P_{i} \oplus S_{i}\right) M\left(P_{i} \oplus S_{i}\right)\right)\right),
$$

with nonzero probability. Since $I \mathbf{1}=\mathbf{1}$, Corollary 4.13 implies that the polynomial on the right hand side is equal to

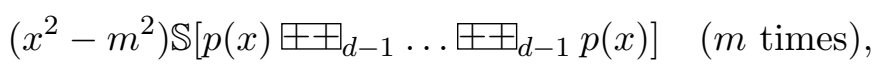

where

$$
p(x)=\chi_{x}\left(I_{d-1} I_{d-1}^{T}\right)=(x-1)^{d-1} .
$$


We upper bound the inverse Cauchy transform of this $m$-wise convolution using Theorem 2.8 .

$$
\mathcal{K}_{\mathbb{S}(p \pm \pm \ldots \pm \boxplus p)}(w) \leq m \cdot \mathcal{K}_{\mathbb{S} p}(w)-\frac{m-1}{w}=m \cdot \mathcal{K}_{\left(x^{2}-1\right)^{d-1}}(w)-\frac{m-1}{w} .
$$

Since

$$
\mathcal{G}_{\left(x^{2}-1\right)^{d-1}}(w)=\frac{x}{x^{2}-1},
$$

this is now identical to the calculation (22), so we obtain again the bound $2 \sqrt{m-1}$. Thus, we conclude that $\lambda_{2}(A) \leq 2 \sqrt{m-1}$ with nonzero probability. Since $A$ is bipartite, its spectrum is symmetric about zero, so we must also have $\lambda_{d-1}(A) \geq-2 \sqrt{m-1}$, whence $A$ is Ramanujan.

\section{References}

[BL06] Yonatan Bilu and Nathan Linial. Lifts, discrepancy and nearly optimal spectral gap*. Combinatorica, 26(5):495-519, 2006.

[BSS12] Joshua Batson, Daniel A Spielman, and Nikhil Srivastava. Twice-Ramanujan sparsifiers. SIAM Journal on Computing, 41(6):1704-1721, 2012.

[Fis08] Steve Fisk. Polynomials, roots, and interlacing. arXiv:math/0612833 [math.CA], 2008.

[Fri08] Joel Friedman. A Proof of Alon's Second Eigenvalue Conjecture and Related Problems. Number 910 in Memoirs of the American Mathematical Society. American Mathematical Society, 2008.

[HLW06] Shlomo Hoory, Nathan Linial, and Avi Wigderson. Expander graphs and their applications. Bulletin of the American Mathematical Society, 43(4):439-561, 2006.

[LPS88] A. Lubotzky, R. Phillips, and P. Sarnak. Ramanujan graphs. Combinatorica, 8(3):261$277,1988$.

[Mar88] G. A. Margulis. Explicit group theoretical constructions of combinatorial schemes and their application to the design of expanders and concentrators. Problems of Information Transmission, 24(1):39-46, July 1988.

[MSS15a] A. Marcus, D. A. Spielman, and N. Srivastava. Finite free convolutions of polynomials. arXiv preprint arXiv:1504.00350, April 2015.

[MSS15b] Adam W. Marcus, Daniel A. Spielman, and Nikhil Srivastava. Interlacing families I: Bipartite Ramanujan graphs of all degrees. Annals of Mathematics, 182, 2015. to appear.

[MSS15c] Adam W. Marcus, Daniel A. Spielman, and Nikhil Srivastava. Interlacing families II: Mixed characteristic polynomials and the Kadison-Singer problem. Annals of Mathematics, 182, 2015. to appear.

[Ni191] A. Nilli. On the second eigenvalue of a graph. Discrete Math, 91:207-210, 1991.

[Pem12] Robin Pemantle. Hyperbolicity and stable polynomials in combinatorics and probability. arXiv preprint arXiv:1210.3231, 2012. 